\title{
Research Paper \\ The Effect of a 6-Week Core Stability Training Program on the Stroke Index and Front Crawl Record of Male Swimmers
}

\author{
Mohsenali Darchini ${ }^{1},{ }^{*}$ Teimor Darzabi $\mathbb{1}^{2}$, Mohsen Mofrad Moghadam³ ${ }^{3}$ Mahdi Nabavinik ${ }^{4}$
}

\begin{abstract}
1. Department of Sport Injuries \& Corrective Exercise, Faculty of Physical Education and Sport Science, Ferdowsi University of Mashhad, Mashhad, Iran. 2. Department of Professional Sciences, Faculty of Mohammad Montazeri, Khorasan Razavi Branch, Technical and Vocational University, Mashhad, Iran. 3. Department of Sport Injuries \& Corrective Exercise, Faculty of Physical Education and Sport Science, Tehran University, Tehran, Iran

4. Department of Motor Behavior, Faculty of Physical Education and Sport Science, Mazandaran University, Mazandaran, Iran.
\end{abstract}

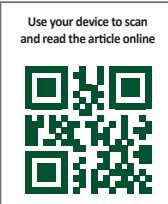

Cttat On: Darchini M, Darzabi T, Mofrad Moghadam M, Nabavinik M. [The Effect of a 6-Week Core Stability Training Program on the Stroke Index and Front Crawl Record of Male Swimmers (Persian)]. Journal of Sport Biomechanics. 2019; 5(2):124133. https://doi.org/10.32598/biomechanics.5.2.6

doi'https://doi.org/10.32598/biomechanics.5.2.6

Key words:

Core stability, Stroke index, Swimming Record

\section{A B STRACT}

Objective For optimal performance of swimmers, it is necessary to determine a level for the functional interaction of upper and lower limbs. This level, created by more than 20 pairs of muscles, is the core of the body. The aim of this study was to investigate the effects of six weeks of core stability exercise on the stroke index and front crawl record of male swimmers.

Methods In this quasi-experimental study, 24 male swimmers were selected by a convenience sampling method and randomly assigned into control $(n=12)$ and exercise $(n=12)$ groups. The core stability training was performed for 6 weeks, 3 sessions per week, each for 45-50 minutes. Before and after training, the stroke index and 100-m front crawl records of subjects were evaluated. For the within-group and between-group comparisons, the student t-test and ANCOVA were used, respectively. The significance level was set at $\mathrm{P}<0.05$.

Results Six weeks of core stability exercise led to significant improvement in 100-m front crawl record in the exercise group. There was no significant difference in the mean scores of stroke index and 100-meter front crawl record between and within the two study groups.

Conclusion Core stability exercise is recommended for dryland training of swimmers.

\section{Extended Abstract}

\section{Introduction}

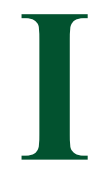

$\mathrm{n}$ recent years, many countries have made great efforts to win medals in various sports competitions, including the Olympic Games and World Championships [1]. Swimming is one of the sports where athletes have won many medals in the Olympic Games. In order to improve front crawl swimming records, it is necessary to perform effective techniques. One of the effective factors in improving the performance of swimmers is the stroke index (stroke length and stroke number). Strong core stability transfers the produced forces of the lower limbs to the upper limbs and improves athletic performance [2]. On the other hand, weak core stability impairs energy transfer and reduces athletic performance [3].

\section{* Corresponding Author:}

Teimour Darzabi, PhD.

Address: Department of Professional Sciences, Faculty of Mohammad Montazeri, Khorasan Razavi Branch, Technical and Vocational University, Mashhad, Iran Tel: +98 (51) 38781015

E-mail: tdarzabi@tvu.ac.ir 
Therefore, it seems appropriate to use regular core stability exercises to reduce injury, improve the record and performance in swimmers. Due to the importance of the subject and the little research done in this field, especially in relation to swimmers, the present study aimed to examine the effect of 6-week core stability exercise on the stroke index and the front crawl swimming record in adolescent elite swimmers.

\section{Participants and Methods}

This is a quasi-experimental applied study with pretest/ posttest design. Study samples were 24 swimmers with a mean age of $12.60 \pm 1.60$ years, mean height of $165.05 \pm 12.54$ $\mathrm{cm}$ and mean weight of $48.90 \pm 10 \mathrm{~kg}$. They were selected using a convenience sampling technique and based on inclusion criteria. No history of tobacco use, no supplementation before participating in the training program and being a member of the national team or the selected team of the province were some of the criteria for entering the study.

Having cardiovascular disease, diabetes, high blood pressure and a history of previous spinal and lower extremity injuries were the exclusion criteria. First, 30 samples completed a health and physical questionnaire (surveying mental and emotional states, the amount of physical activity per day, and problems during physical activity), and after analyzing the questionnaire, 24 were selected to participate in the study. Samples voluntarily participated in the study and signed a consent form. They were then randomly divided into exercise $(n=12)$ and control $(n=12)$ groups.

The core stability training program was performed for 6 weeks, 3 sessions per week, each for 45-50 minutes. It included 15-min warm-up, aerobic exercise for 10-25 minutes, and 10-min cooling down. Warming up session included stretching movement and general warming of the joints. The intensity of the training gradually increased from the first to the 5th week and decreased in the last week due to the participation of subjects in the functional tests. The training program was performed on both core stability muscles including small (muscles that attach to the lumbar vertebrae and affect intercostal movements) and large muscles (those attach to the pelvis and hip).

On the first day and after a special warm-up, swimmers were asked to swim 100 meters breaststroke in a 25 -meterlong pool with all their might to estimate the distance of the stroke and the frequency of the stroke. On the first day and after a special warm-up, swimmers were asked to perform 100 meter front crawl in a 25 -meter-long pool with all their power to estimate stroke rate and distance per stroke. Each subject was tested three times and the best record and the best stroke number were recorded. The test taker counted and recorded the number of strokes by moving along the pool. At the end of the swim, the best time was considered as the swimmer's record.

At the end of each session, the cooling down was performed by stretching and relaxation movements. During this time, the subjects in the control group performed their routine exercises. At the end of intervention and after one day of rest, the tests were performed in both groups. The collected data were analyzed in SPSS v.16 software. After confirming the normality of data distribution using the Shapiro-Wilk test and the homogeneity of the variances by Levene's test, student t-test was sued for to intergroup comparison and ANCOVA for intergroup comparison of mean changes. The significance level was set at $\mathrm{P}<0.05$.

\section{Results}

Based on the results presented in Table 1, the t-test results showed that the 6-week core stability training program led to a significant improvement in the subjects' 100 -meter front crawl record $(\mathrm{P}=0.02)$, but there was no significant

Table 1. Comparing the mean scores of stroke index and front crawl record between and within groups

\begin{tabular}{|c|c|c|c|c|c|c|c|}
\hline \multirow{3}{*}{ Variables } & \multirow{3}{*}{ Group } & \multicolumn{2}{|c|}{ Mean $\pm S D$} & \multicolumn{4}{|c|}{ Difference } \\
\hline & & \multirow{2}{*}{ Pre-test } & \multirow{2}{*}{ post-test } & \multicolumn{2}{|c|}{ Within-Group } & \multicolumn{2}{|c|}{ Between-Group } \\
\hline & & & & $\mathbf{T}$ & $\mathbf{P}$ & $\mathbf{F}$ & $\mathbf{P}$ \\
\hline \multirow{3}{*}{ stroke index } & Exercise & $66 \pm 8.95$ & $11 \pm 68.37$ & 0.825 & 0.4 & \multirow{3}{*}{0.73} & \multirow{3}{*}{0.4} \\
\hline & & & & & & & \\
\hline & Control & $66.7 \pm 5.99$ & $71.5 \pm 8.4$ & 2.188 & 0.06 & & \\
\hline \multirow{3}{*}{ 100-m front crawl record } & Exercise & $120.12 \pm 6.7$ & $110.9 \pm 5.4$ & 4.077 & $0.02 *$ & \multirow{3}{*}{0.73} & \multirow{3}{*}{0.39} \\
\hline & & & & & & & \\
\hline & Control & $114 \pm 12.7$ & $115.0 \pm 15.5$ & 0.349 & 0.73 & & \\
\hline
\end{tabular}


change in their stroke index $(\mathrm{P}=0.4)$. In the control group, no significant changes were observed in subjects' front crawl record and stroke index. According to the results in Table 1, the ANCOVA results showed no significant difference in the mean scores of stroke index $(\mathrm{P}=0.4)$ and 100 -meter front crawl record $(\mathrm{P}=0.39)$ between and within the two study groups.

\section{Conclusion}

Core stability training can improve swimmers' front crawl records; so, it is recommended that these exercises be included in the swimmers' dryland training. Moreover, due to the lack and contradiction of the results regarding the effect of core stability exercises on the performance of swimmers, more studies are recommended in this area.

\section{Ethical Considerations}

Compliance with ethical guidelines

All ethical principles were considered in this study. The subjects were free to leave the study at any time, and were assured of the confidentiality of their information.

\section{Funding}

This research did not receive any specific grant from funding agencies in the public, commercial, or not-forprofit sectors.

\section{Authors' contributions}

All authors contributed equally in preparing this article.

\section{Conflicts of interest}

The authors declared no conflict of interest. 


\title{
تغييرات شاخص دست وركورد شناى كرال سينه شناتر ان به دنبال شش هفته تمرين ثبات مركزى
}

\author{
محسنعلى دارجينى'، "ثيمور درزابى "ه، محسن مفرد مقدم"، مهدى نبوى نيى"
}

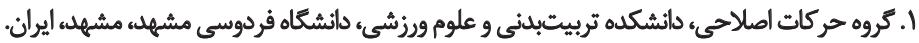

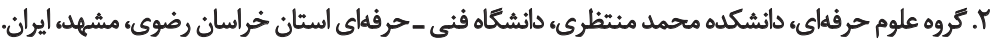

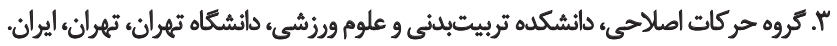

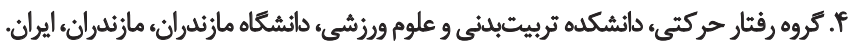

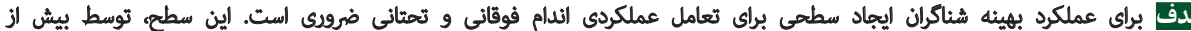

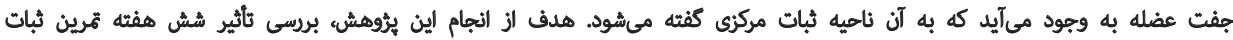
مركزى بر شاغص دست و وكورد شناي كرال سينه شناكران بود.

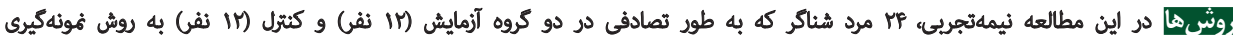

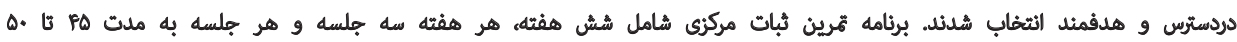

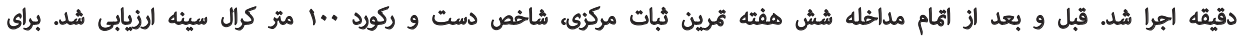

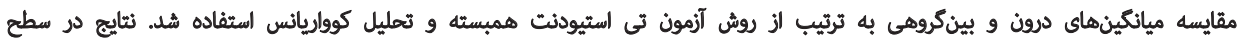

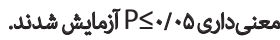

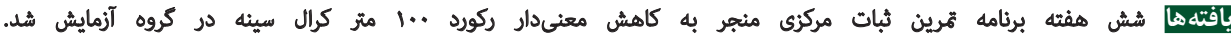

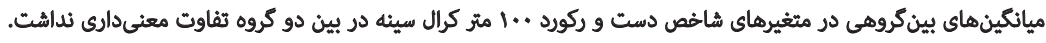

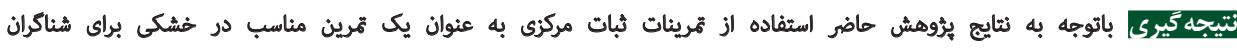

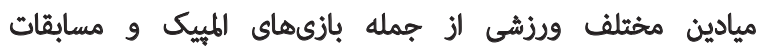

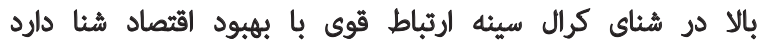

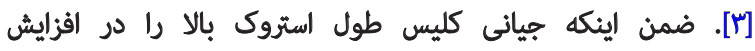

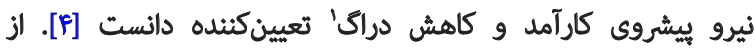

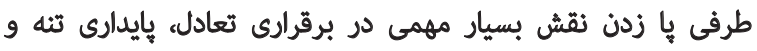

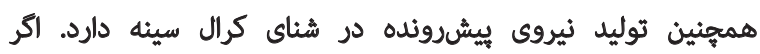

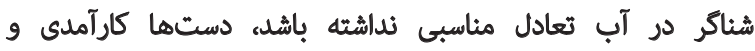
تأثيركذارى كمترى خواهند داشت [هاد]. سورت ول كزارش كرد، طول استروك (مسافت طى شده با هر

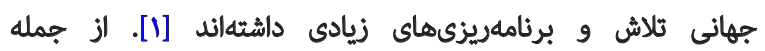

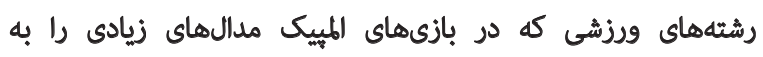

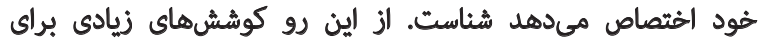

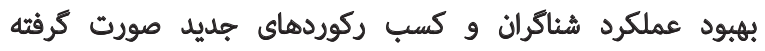

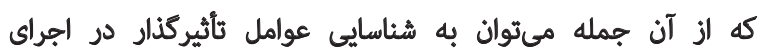

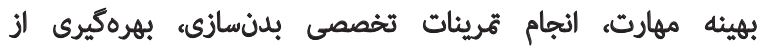

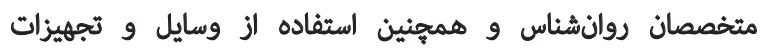

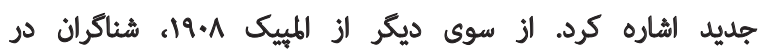

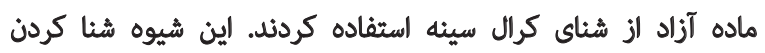

$$
\text { …… }
$$

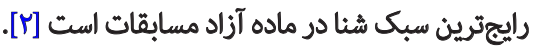




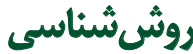

اين تهقيق الز نوع كاربردى نيمهتجربى است كه با طرح

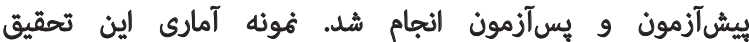

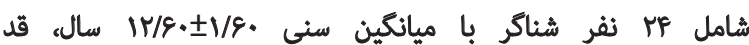

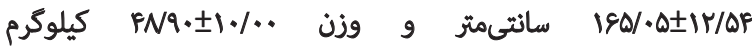

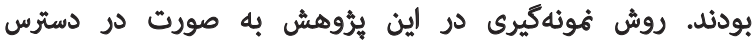

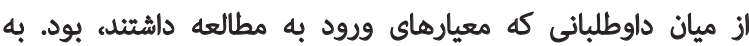
منظور رعايت ملاحظات الخلاقى، رضايتنامه آكاهانهاى به ديه دنبال

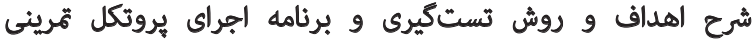

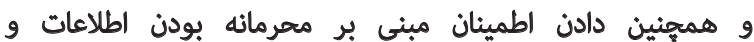

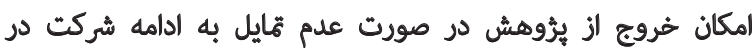

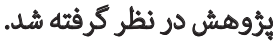

نخست افراد با ماهيت و نحوه همكارى با اجراى برُوهش آشنا

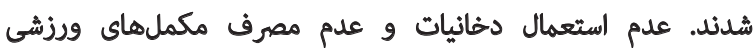

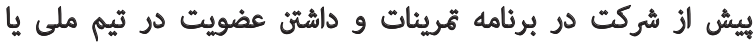

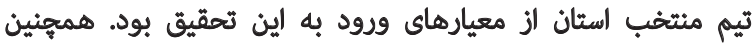

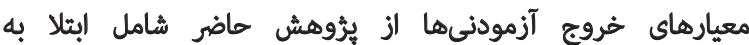

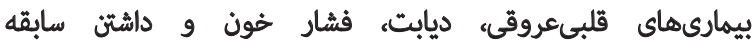

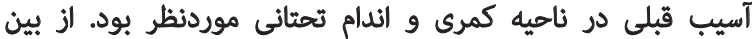

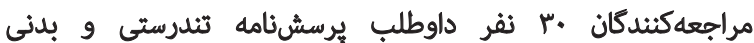

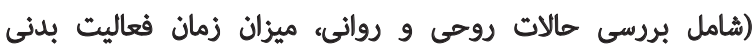

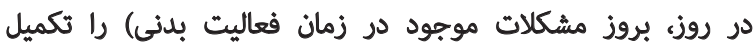

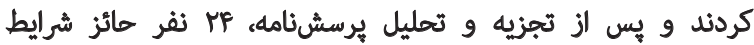

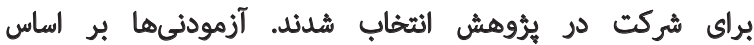

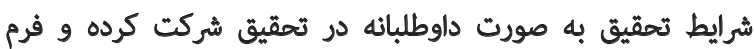

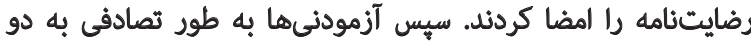
كروه آزمايش (rا نف) و كنترل (rا نفر) تقسيم شدند.

$$
\text { اندازهيَيرى عملكردشنائران }
$$

در اين تهقيق برايى ارزيابى عملكرد شناكران از زمان شنا و ثواتر

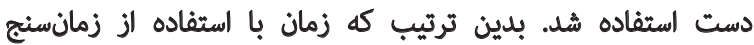

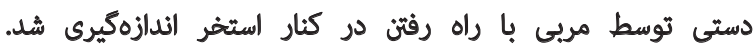

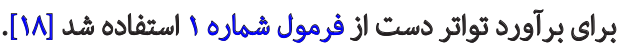

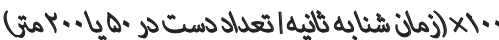

در اين تحقيق فعاليت ورزشى منظم، برنامه تمرينات ثبات

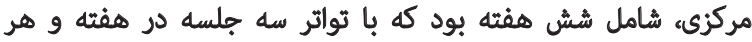

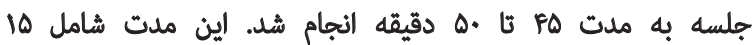

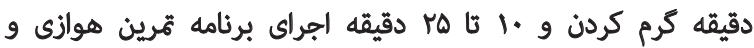

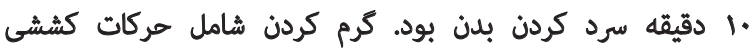

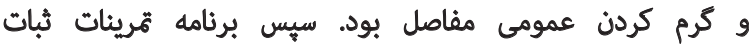

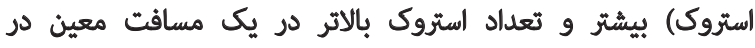

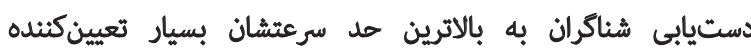

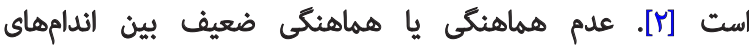

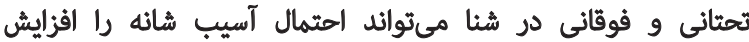

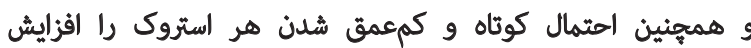

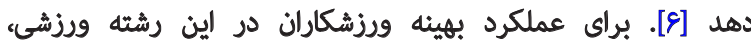

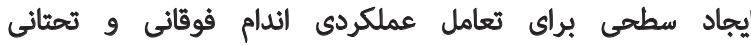

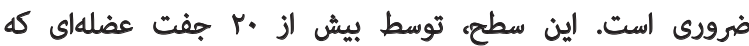

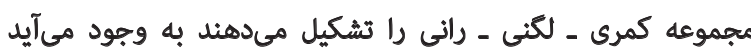

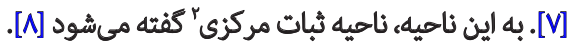

كيبلر و همكاران ناحيه ثبات مركزى را در فعاليتهاى ورزشى ئى

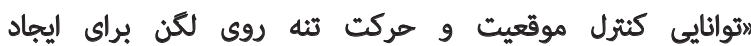

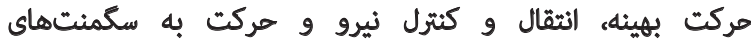

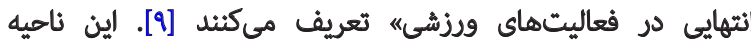

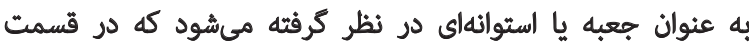

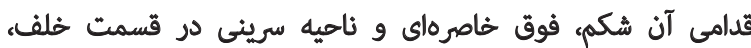

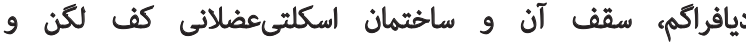

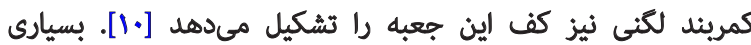

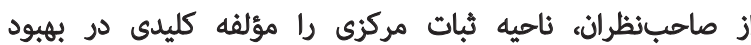

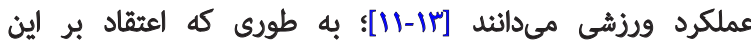

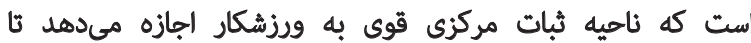

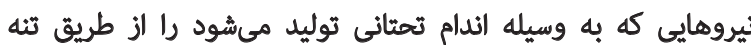

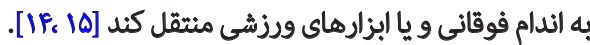
از طرفى نسر و لى بيان كردند كه ناحيه ثبات مركزى ضعيف،

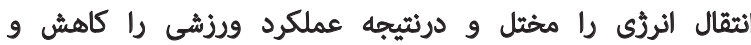

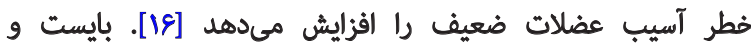

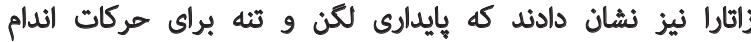

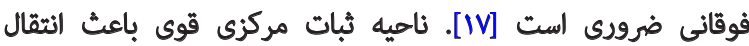

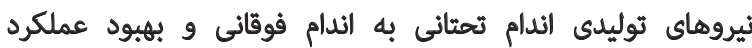

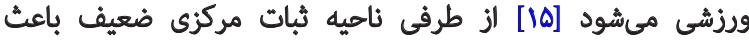

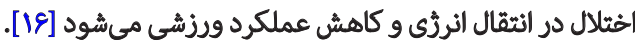
بر اساس اين اطلاعات به نظر مىرسد استفاده از تمرينات

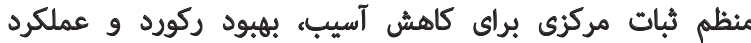

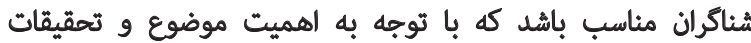

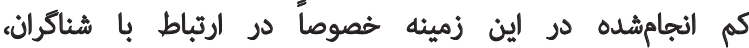

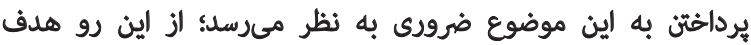

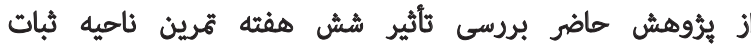

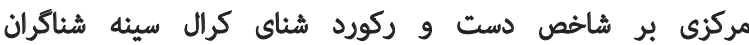

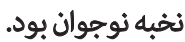

2. Core stability 
بر اساس يافتههاي جدول شماره ب، نثايج تي وابسته نشان داد

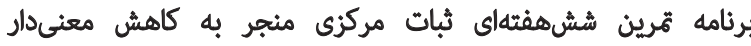

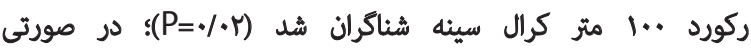

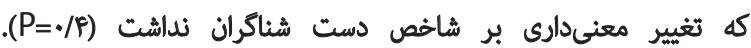

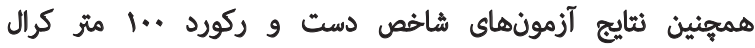

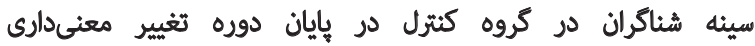

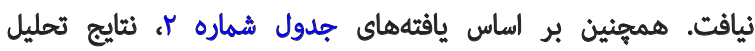
كوواريانس نشان داد تغييرات ميانكينهاى بين كروهى در مر متغيرهاي

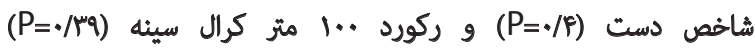
تفاوت معنى ارى بين دو كروه آزمايش و كنترل مشاهده نشد.

40

هدف الز يُوهش حاضر، برزسى ثأثير يك دوره برنامه ثرينى

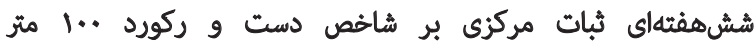

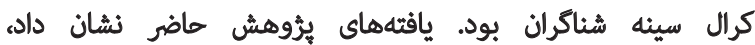

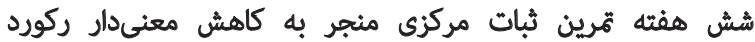

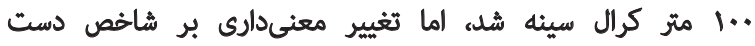

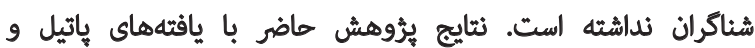

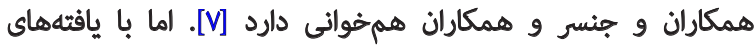

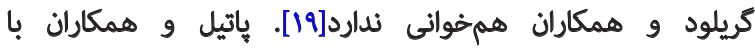

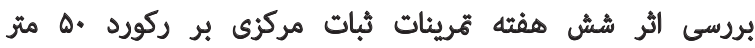

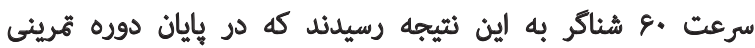

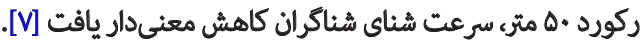

جنسر و همكاران با بررسى مقايسه اثر هشت هفته ثمرينات

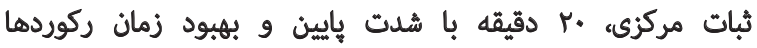

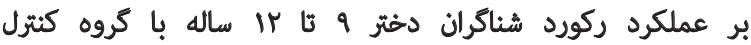

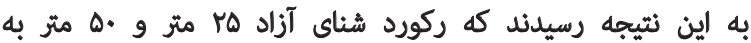

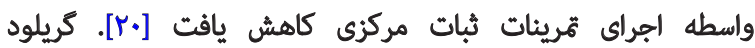

مركزى كه براي تقويت ثاهيه ثبات مركزى طراهى شده بوده الجرا

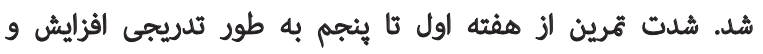

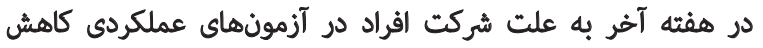

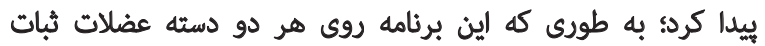

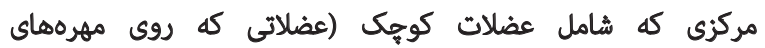
كمرى مى مرسبند و بر حركات بين بخشى مؤثر هستند) و عضلات

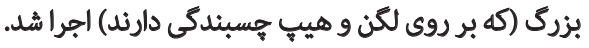

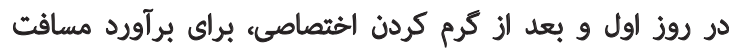

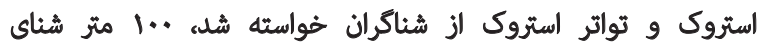
كرال سينه را در استخرى با طول ها مت متر با تمام ثوان شئنا كنئ.

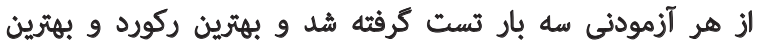

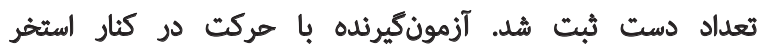

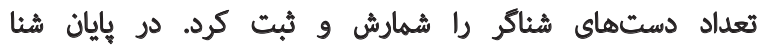

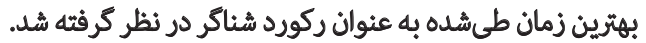

در انتهاى هر جلسه تمرين عمل سرد كردن و بركشت به به

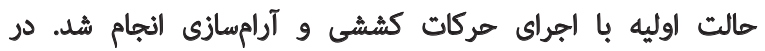

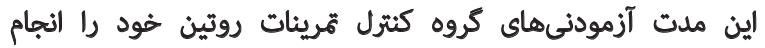

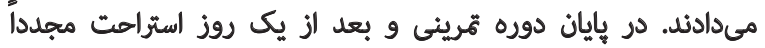
آزمونهاي موردنظر از شناكران هر دو كروه به دمل دمل آمد.

در يايان دادههاى جمعآورىشده با كمك نرم افزار SPSS ويرايش

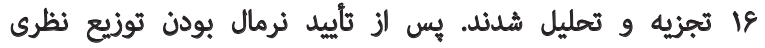

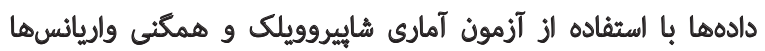

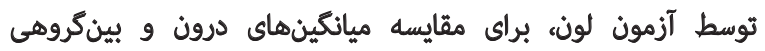

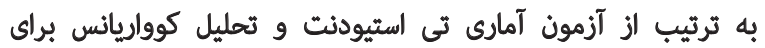

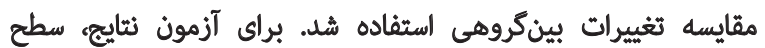

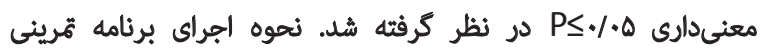

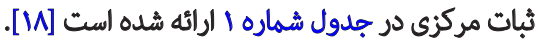

جدول ا. برنامه يُشرونده كروه تمرين ثبات مركزى در خشكى

\begin{tabular}{|c|c|c|c|c|c|c|c|}
\hline هفته ششمي & هفته بنتجمي & هفته جهارم & هفته سوم & هفته دوم & هفته اول & اضضافه بار & تمرين \\
\hline 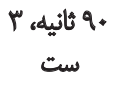 & 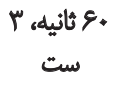 & 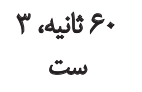 & 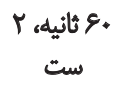 & مثت مثانيه، & "ب ثانيه، ب & حجم & ميلانك (يل زدن از جلو) \\
\hline 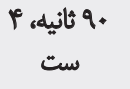 & 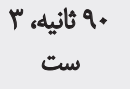 & 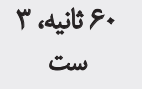 & 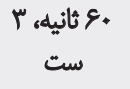 & كائيه، & 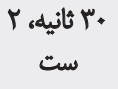 & حجم & يلانك جائب (يل زدن از جانب روى آرئج) \\
\hline هو ثانيه، F & مبت مثائه، & 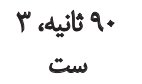 & 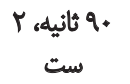 & ع ثائيه، & كثت بانيه، & حجم & سك يرنلده \\
\hline معت مائيه. & مت نانيه، & مثت بائيه، & 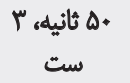 & "r ثائيه، ب. & ست باثيه. & حجمر & يازدن فلاتر (Flutter kick) \\
\hline
\end{tabular}

مجله بيومكانيك وزنش 
جدول r. ميانكين و انحراف استائدارد تغييرات درون و بين كروه متغيرهاي تحقيق

\begin{tabular}{|c|c|c|c|c|c|c|c|}
\hline \multicolumn{4}{|c|}{ تغييرات } & \multicolumn{2}{|c|}{ ميانكين士 انحراف معيار } & \multirow{3}{*}{ كروه } & \multirow{3}{*}{ متغير } \\
\hline \multicolumn{2}{|c|}{ بين كروهي } & \multicolumn{2}{|c|}{ ورون كروه } & \multirow{2}{*}{ بس آزمون } & \multirow{2}{*}{ ييش آزمون } & & \\
\hline $\mathbf{P}$ & $\mathbf{F}$ & $\mathbf{P}$ & $\mathbf{T}$ & & & & \\
\hline \multirow{2}{*}{$\cdot / p$} & \multirow{2}{*}{$\cdot M$} &.$/ 4$ & - IATA & FA土II/Tr & $\Leftrightarrow \pm N Q \Delta$ & تجريى & \multirow{2}{*}{ شاخص دست } \\
\hline & & .1 .8 & $r / M M$ & $V I / \Delta \pm N F$ & $8 \& / N \pm \Delta / 9 q$ & كنترل & \\
\hline \multirow{2}{*}{.$/ 1 \% q$} & \multirow{2}{*}{$\cdot / N^{n}$} & $.1+Y^{*}$ & $p / \cdot W$ & $11 \cdot / \Delta \pm \sqrt{ } / 4$ & $I T \cdot 1 F \pm I T / N$ & تجربى & \multirow{2}{*}{ ركورد +.ا متر كرال سينه } \\
\hline & & $\cdot M^{N}$ &.$/ 4 r q$ & $11 \phi / \cdot \pm 1 \theta / \Delta$ & $\| F \pm I F / Y$ & كنترل & \\
\hline
\end{tabular}

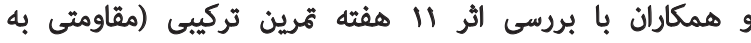

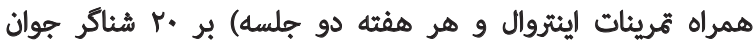

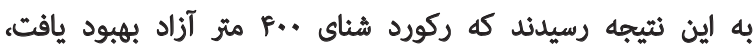

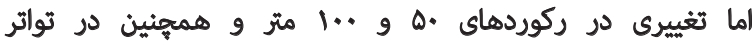

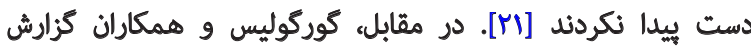

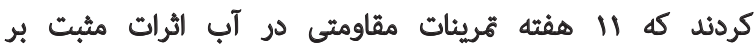

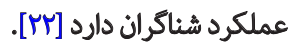

با توجه به اينكه عنوان شده كه تواتر دست مهمترين شاخص

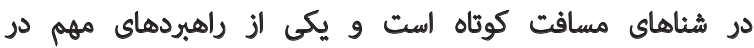

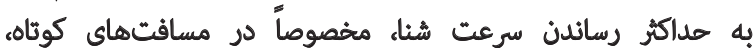

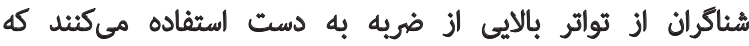

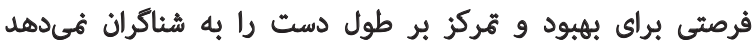

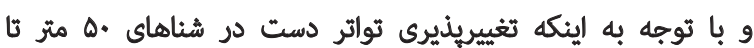

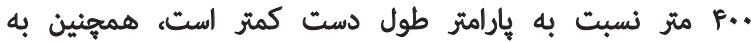

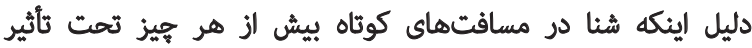

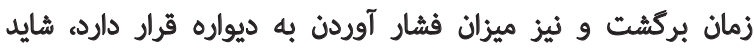

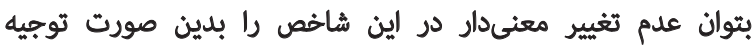
كرد [IN

در اين زميئه، بر اساس نثايج مطالعات بين عملكرد شئاى كرال

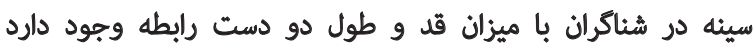

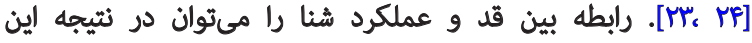

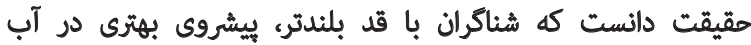

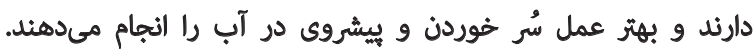

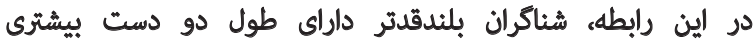

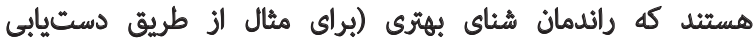

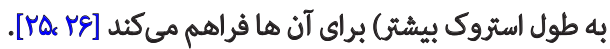

\section{نتيجلكيرى نهايى}

يافتهان اين هُروهش نشان داد كه ثمرينات ثبات مركزى

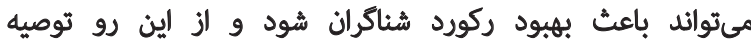

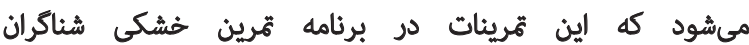

و همكاران كزارش كردند كه خشكى و آب منجر به عدم تغيير معنى الارى در عملكرد شناكران

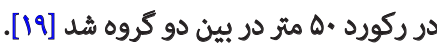

يكى از دلايل نتايج متناقض در يُروهش كريلود و همكاران

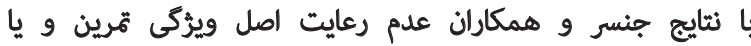

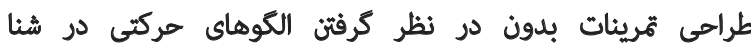

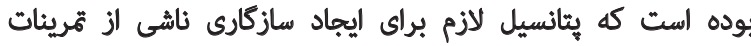

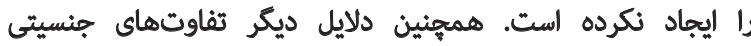

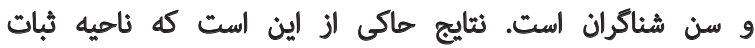

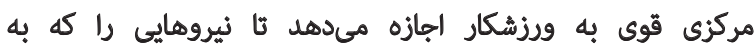

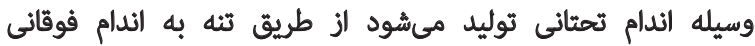

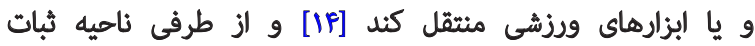

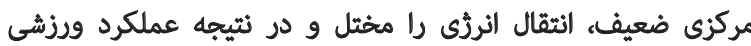

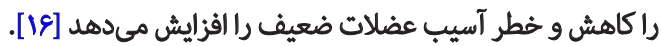

با توجه به نثايج و اينكه در فاكثور شاخص دست شناكران

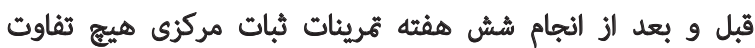

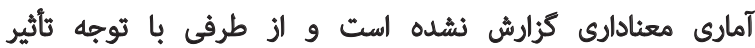

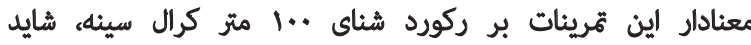

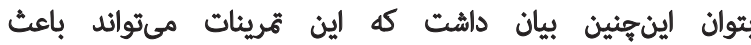

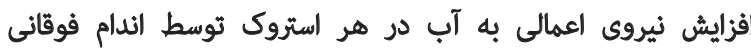

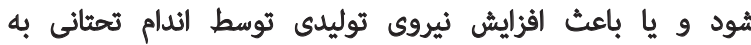

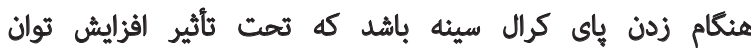

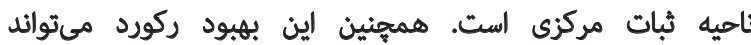

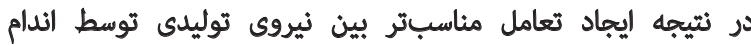

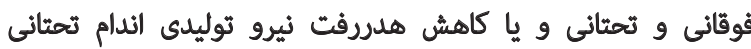
در ناحيه ثبات مركزى با توجه به افزايش ثواني كوان اين ناحيه و انثقال

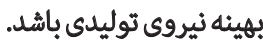

يافتهاى يُروهش حاضر نشان داد، شُش هفته تمرين ثبات

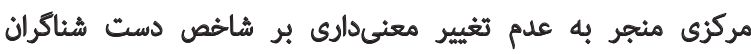

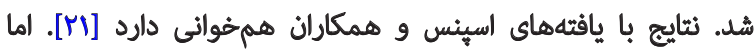
با يافتهاى كوركوليس و همكاران همخواني ندارد [بر]. اسينس 
كنجائده شود. همجين به دليل كمبود و ضد و نقيض بودند

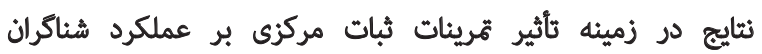
تحقيقات بيشتر در اين زمينه ييشئهاد مئشود.

\section{ماحظات اخلاقى}

$$
\text { ييروى از اصول اخلاق يثوهش }
$$

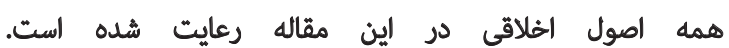

شركتكنندكان الجازه داشتئ هر زمان كه مايل بودند الز ئزوهش

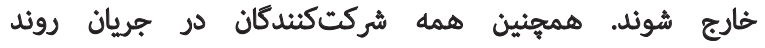
يُروهش بودند و اطلاعات آنها محرمائه نكه دالشته شد. شمد.

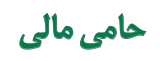

اين بؤوهش هيجكوثه كمك مالى از سازمانهاى دولتى، هصوصى و غير اثتثفاعى دريافت نكرده استيت.

$$
\text { مشاركت نويسند متان }
$$

قمام نويسندكان در آمادهسازى اين مقاله مشاركت داشتهاند.

$$
\text { تعارض مناقع }
$$

بنابر اظهار نويسندكان اين مقاله تعرض منافع ندارد. 


\section{References}

[1] Vaeyens R, Güllich A, Warr CR, Philippaerts R. Talent identification and promotion programmes of Olympic athletes. Journal of sports sciences. 2009;27(13):1367-80. [DOI:10.1080/02640410903110974] [PMID]

[2] Sortwell AD. Relationship between stroking parameters and leg movement quantity in 100 metre front crawl. International Journal of Exercise Science. 2011; 4(1):22-9. [PMID] [PMCID]

[3] Costill DL, Kovaleski J, Porter D, Kirwan J, Fielding R, King D. Energy expenditure during front crawl swimming: Predicting success in middle-distance events. International Journal of Sports Medicine. 1985; 6(5):266-70. [DOI:10.1055/s-2008-1025849] [PMID]

[4] Sanders R. New analysis procedures for giving feedback to swimming coaches and swimmers. Paper presented at: XX ISBS-Swimming, Applied Program Swimming. 1-5 July 2002; Cáceres, Spain. https://www. researchgate.net/publication/325810276

[5] Toussaint HM, Van Den Berg C, Beek WJ. "Pumped-up propulsion" during front crawl swimming. Medicine and Science in Sports and Exercise. 2002; 34(2):314-9. [DOI:10.1097/00005768-200202000-00020] [PMID]

[6] Toussaint HM, Beek PJ. Biomechanics of competitive front crawl swimming. Sports Medicine. 1992; 13:8-24. [DOI:10.2165/00007256199213010-00002]

[7] Patil D, Salian SC, Yardi S. The effect of core strengthening on performance of young competitive swimmers. International Journal of Science and Research. 2014; 3(6):2470-7. https://www.researchgate.net/publication/269108164

[8] Karpiński J, Rejdych W, Brzozowska D, Gołaś A, Sadowski W, Swinarew AS, et al. The effects of a 6-week core exercises on swimming performance of national level swimmers. BioRxiv. 2019 December. https:// www.biorxiv.org/content/10.1101/2019.12.19.882126v1.full

[9] Kibler WB, Press J, Sciascia A. The role of core stability in athletic function. Sports Medicine. 2006; 36(3):189-98. [DOI:10.2165/00007256200636030-00001] [PMID]

[10] Richardson C, Jull G, Hodges P, Hides J. Therapeutic exercise for spinal segmental stabilization in low back pain: Scientific basis and clinical approach. London: Churchill Livingstone; 1999. https://books.google.com/ books?id= gXdPgAACAAJ\&dq

[11] McGill SM. Low back stability: From formal description to issues for performance and rehabilitation. Exercise and Sport Sciences Reviews. 2001; 29(1):26-31. [DOI:10.1097/00003677-200101000-00006] [PMID]

[12] Leetun DT, Ireland ML, Willson JD, Ballantyne BT, Davis IM. Core stability measures as risk factors for lower extremity injury in athletes. Medicine \& Science in Sports \& Exercise. 2004; 36(6):926-34. [DOI:10.1249/01. MSS.0000128145.75199.C3] [PMID]

[13] Jeffreys I. Developing a progressive core stability program. Strength \& Conditioning Journal. 2002; 24(5):65-6. [DOI:10.1519/00126548200210000-00017]

[14] Behm DG, Leonard AM, Young WB, Bonsey WA, MacKinnon SN. Trunk muscle electromyographic activity with unstable and unilateral exercises. Journal of Strength and Conditioning Research. 2005; 19(1):193-201. [DOI:10.1519/1533-4287(2005)19<193:tmeawu>2.0.co;2] [PMID]

[15] Cissik JM. Programming abdominal training, Part II. Strength and Conditioning Journal. 2002; 24(2):9-12. [DOI:10.1519/00126548200204000-00002]

[16] Nesser TW, Lee WL. The relationship between core strength and performance in division I female soccer players. Journal of Exercise Physi- ology Online. 2009; 12(2):21-8. https://www.researchgate.net/publication/228494628

[17] Bouisset S, Zattara M. A sequence of postural movements precedes voluntary movement. Neuroscience Letters. 1981; 22(3):263-70. [DOI:10.1016/0304-3940(81)90117-8]

[18] Harati J, Daneshmandi H, Shahabi Kaseb MR. [Comparing the effects of dry-land and in-water core stability training programs on swimmers' upper body balance and performance (Persian)]. Journal of Sport Biomechanics. 2018; 4(1):17-29. http://biomechanics.iauh.ac.ir/article1-152-en.html

[19] Girold S, Maurin D, Dugué B, Chatard JC, Millet G. Effects of dry-land vs. resisted-and assisted-sprint exercises on swimming sprint performances. Journal of Strength and Conditioning Research. 2007; 21(2):599-605. [DOI:10.1519/00124278-200705000-00054] [PMID]

[20] Gencer YG. Effects of 8-week core exercises on free style swimming performance of female swimmers aged 9-12. Asian Journal of Education and Training. 2018; 4(3):182-5. [DOI:10.20448/jourf nal.522.2018.43.182.185]

[21] Aspenes S, Kjendlie PL, Hoff J, Helgerud J. Combined strength and endurance training in competitive swimmers. Journal of Sports Science \& Medicine. 2009; 8(3):357-65. [PMID] [PMCID]

[22] Gourgoulis V, Valkoumas I, Boli A, Aggeloussis N, Antoniou P. Effect of an 11-week in-water training program with increased resistance on the swimming performance and the basic kinematic characteristics of the front crawl stroke. The Journal of Strength \& Conditioning Research 2019; 33(1):95-103. [DOI:10.1519/JSC.0000000000001879] [PMID]

[23] Lätt E, Jürimäe J, Mäestu J, Purge P, Rämson R, Haljaste K, et al. Physiological, biomechanical and anthropometrical predictors of sprint swimming performance in adolescent swimmers. Journal of Sports Science \& Medicine. 2010; 9(3):398-404. [PMID] [PMCID]

[24] Silva AJ, Costa AM, Oliveira PM, Reis VM, Saavedra J, Perl J, et al The use of neural network technology to model swimming performance. Journal of Sports Science \& Medicine. 2007; 6(1):117-25. [PMID] [PMCID]

[25] Saavedra JM, Escalante Y, Rodríguez FA. A multivariate analysis of performance in young swimmers. Pediatric Exercise Science. 2010; 22(1):135-51. [DOI:10.1123/pes.22.1.135] [PMID]

[26] Geladas ND, Nassis GP, Pavlicevic S. Somatic and physical traits affecting sprint swimming performance in young swimmers. International Journal of Sports Medicine. 2005; 26(2):139-44. [DOI:10.1055/s-2004-817862] [PMID] 
This Page Intentionally Left Blank 